\title{
The possibility of an appropriate neutron beam achievement for medical purposes based on GEANT4 calculations
}

\author{
V. Ivanyan ${ }^{\mathrm{a}}$ \\ Faculty of Physics, Astronomy and Applied Informatics, Jagiellonian University in Krakow, Krakow, Poland
}

Received: 21 March 2019 / Accepted: 3 January 2020 / Published online: 13 January 2020

(C) The Author(s) 2020

\begin{abstract}
Proton-induced reactions on the ${ }^{9}$ Be target are considered as a neutron source, and dependence of neutron yield on target thicknesses is investigated. The optimal thickness of the ${ }^{9} \mathrm{Be}$ target with the design and optimization characteristics of a beam shaping assembly (BSA) for neutron flux from the thick target is studied with the GEANT4 program. To create the realistic model of the experiment, there are inserted two physics lists for nuclear and electromagnetic reactions. To get a high flux of neutrons had taken into account usage of special materials as moderators on the base of $(n, 2 n)$ nuclear reactions, as well as, moderators which will decrease the energy of neutrons to achieve the appropriate thermal/epithermal neutron flux. The creation of the system, which must be made from reflectors and moderators, is a necessity to explore the possibility of an appropriate neutron flux achievement for medical purposes, especially for boron neutron capture therapy (BNCT). GEANT4 simulations of this scientific paper describe the study of IBA's C18/18 cyclotron-based neutron sources and its possible usage for therapies.
\end{abstract}

\section{Introduction}

An ability of ${ }^{10} \mathrm{~B}$ nuclei to capture thermal neutrons was shown first time by H.J. Taylor, where the capture process has resulted in the nuclear fission of the ${ }^{11} \mathrm{~B}$ nuclei into ${ }^{4} \mathrm{He}$ (alpha particles) and ${ }^{7} \mathrm{Li}$ ions. Right after this discovery, G.L. Locher suggested that the neutron capture process could be used to treat cancer. Later it was called boron neutron capture therapy (BNCT) and became a promising method of treatment of malignant tumors. Briefly summarized, it is a two-step procedure: first, the patient is injected with a tumor-localizing drug containing the non-radioactive isotope ${ }^{10} \mathrm{~B}$ that has a high propensity or cross section to capture slow neutrons. In the second step, the patient is radiated with thermal/epithermal neutrons. After the loss of energy during passage through tissue, neutrons are absorbed by the capture agent, which subsequently emits alpha particles. These charged particles can selectively kill tumor cells in which a sufficient amount of ${ }^{10} \mathrm{~B}$ is concentrated.

Therapeutic interest in BNCT has focused mainly on high-grade gliomas (brain tumors) [1-3], and more recently on patients with recurrent tumors of the head and neck region [4-8] who have failed conventional therapy. BNCT primarily is a biochemically rather than a

a e-mail: vahag.ivanyan@gmail.com 
physically targeted type of radiation therapy, and, therefore, it should be possible to selectively destroy tumor cell by cell, providing that sufficient amounts of ${ }^{10} \mathrm{~B}$ and thermal/epithermal neutrons are delivered to the site of the tumor.

To date, almost all BNCT clinical irradiation have used fission reactor neutron sources, because of the appropriate neutron flux achievement; however accelerator-based neutron sources are under development and may eventually provide another option to fission reactors. Accelerator-based neutron sources are being developed with the anticipation of being easier to site in hospitals and license than a dedicated nuclear reactor. Also, they could be more compact and less expensive than comparable reactor sources. Generally, they produce low-intensity neutron fluxes compared to reactor sources and this has been a problem for implementation. An increase in intensity by more than an order of magnitude would be necessary if accelerators are to be competitive with the better reactor sources for clinical BNCT.

Newly installed Cyclone 18/18 in the area of the Armenian National Science Laboratory after A. Alikhanian has a possibility to produce $18 \mathrm{MeV}$ proton beams with up to $100 \mu \mathrm{A}$ current, which is approximately $6.24 \times 10^{14}$ proton/s. The modified cyclotron from IBA has a $4 \mathrm{~m}$ vacuum pipe for passing external protons to the experimental hall, and the beam spot on target is $10 \mathrm{~mm}[9,10]$. The aluminum foil with $500 \mu \mathrm{m}$ thickness is installed right on the end of the pipe as vacuum keeping window. The presence of the aluminum foil decreases the initial beam energy from 18 to $14.8 \mathrm{MeV}$.

The complicated processes connected with the selection of the effective target with optimal thickness, and the creation of the beam shaping assembly (BSA) was studied by GEANT4 program, to understand the possibility of the appropriate neutron flux achievement and its usage in BNCT. BSA is needed to optimize the initial neutron flux and during the research, were taken into account the usage and selection of those materials, which has possibilities to multiply neutrons, and as result increase the flux and at the same decrease the energy. The continuation of the optimal flux achievement should be followed by installing filtering materials for thermal and fast neutrons. In the end, the fast and thermal components of the final flux should be much less than the epithermal component, but the total flux should be in the order of $\sim 10^{9} \mathrm{n} / \mathrm{s} / \mathrm{cm}^{2}[11,12]$. As recommended by the international atomic energy agency (IAEA), the number of epithermal neutrons in the BNCT applicable neutron flux should be $\sim 100$ times more than thermal neutrons, and 20 times more than fast neutrons.

\section{Creation of the GEANT4 model}

GEANT4 is the successor of the GEANT series of software toolkit developed by CERN. To build a specific application, the user-physicist chooses from among the options given by the program and implements code in user action classes supplied by the toolkit. In contrast, object-oriented methods help manage complexity and limit dependencies by defining a uniform interface and common organizational principles for all physics models [13]. Besides other parameters, inside the GEANT4 model of the current feasibility study were used G4HadronPhysicsQGSP_BERT_HP and G4EmStandardPhysics physics lists, responsible for electromagnetic and hadronic processes.

To obtain as high as possible neutron yield, a thick ${ }^{9} \mathrm{Be}$ target has been used. It is installed at a $3 \mathrm{~cm}$ distance after the vacuum pipe and has a $5 \mathrm{~mm}$ radius. All the channels of nuclear reactions, leading to the emission of neutrons, were considered in GEANT4 calculations. The ${ }^{9} \mathrm{Be}(\mathrm{p}, \mathrm{xn})$ multiparticle reactions with thresholds less than $14.8 \mathrm{MeV}$ are listed in Table 1. Figure 1 is a GEANT4 calculation result [14], performed to estimate the optimal and effective thickness of the target. Figure 1a presents neutron energy spectra for different thicknesses 
Table 1 The ${ }^{9} \mathrm{Be}(\mathrm{p}, \mathrm{xn})$ reactions with less than $14.8 \mathrm{MeV}$ thresholds

\begin{tabular}{lll}
\hline Reaction & $Q$ value $(\mathrm{MeV})$ & Reaction threshold $(\mathrm{MeV})$ \\
\hline $\mathrm{p}+{ }^{9} \mathrm{Be} \rightarrow{ }^{9} \mathrm{~B}+n$ & -1.8504 & 2.0572 \\
$\mathrm{p}+{ }^{9} \mathrm{Be} \rightarrow{ }^{9} \mathrm{~B}+n+\gamma$ & -1.8855 & 2.057 \\
$\mathrm{p}+{ }^{9} \mathrm{Be} \rightarrow{ }^{8} \mathrm{Be}+\mathrm{p}+n$ & -1.6645 & 1.8507 \\
$\mathrm{p}+{ }^{9} \mathrm{Be} \rightarrow{ }^{8} \mathrm{Be}+\mathrm{p}+n+\gamma$ & -1.7011 & 1.8507 \\
$\mathrm{p}+{ }^{9} \mathrm{Be} \rightarrow{ }^{5} \mathrm{Li}+\alpha+n+\gamma$ & -3.5377 & 3.9333 \\
$\mathrm{p}+{ }^{9} \mathrm{Be} \rightarrow 2 \alpha+\mathrm{p}+n$ & -1.5727 & 1.74859 \\
\hline
\end{tabular}

(a)

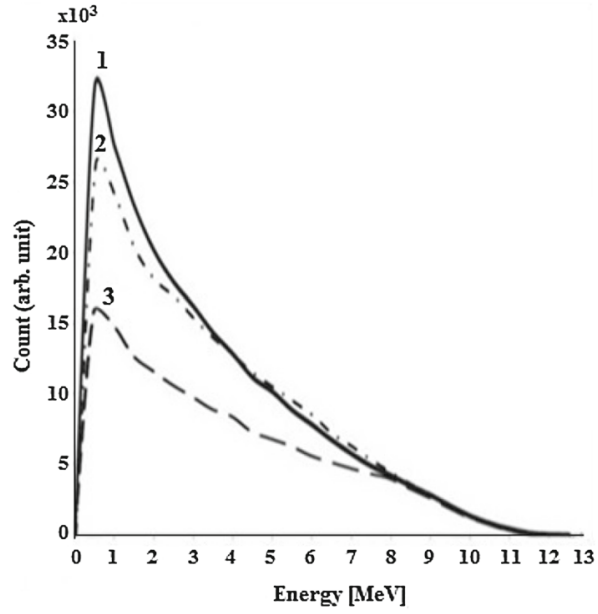

(b)

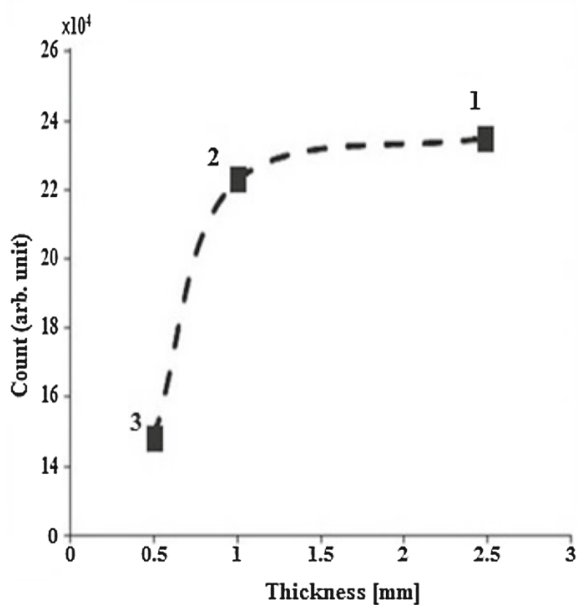

Fig. 1 a Energy spectra of neutrons for $2.5 \mathrm{~mm}(1), 1 \mathrm{~mm}$ (2) and $0.5 \mathrm{~mm}$ (3) thicknesses of ${ }^{9}$ Be target, b Neutron yield dependence from the ${ }^{9}$ Be target thicknesses

of the target $(2.5 \mathrm{~mm}, 1.5 \mathrm{~mm}$, and $0.5 \mathrm{~mm})$, and Fig. $1 \mathrm{~b}$ is the dependence of neutron yield from the different thicknesses. The maximal yield of neutrons was possible to achieve in the case of $2.5 \mathrm{~mm}$ thickness of ${ }^{9} \mathrm{Be}$ target, as proton beam fully absorbed by the target.

The $2.5 \mathrm{~mm}$ thickness is selected as an optimal thickness of the target, to produce as high as possible yield of neutrons. In the case of the thicker target, emitted secondary neutrons will be captured, which will reduce neutron yield. Nevertheless, neutrons from ${ }^{9} \mathrm{Be}(\mathrm{p}, \mathrm{xn})$ reaction source cannot be directly used in BNCT, due to the wide range energy distribution, which reaches up to $13 \mathrm{MeV}$. The final spectrum must show a peak of a quasi-monochromatic neutron, as close as possible to the thermal/epithermal region to make possible neutron capture processes by boron-10. Based on the number of registered neutrons in the energy range up to a few $\mathrm{keV}$, it could be realistic, the usage of the neutron flux for BNCT. The acceleratorbased neutron sources (ANS) should be comparable with reactor-based neutron sources, and usually, there is a necessity to increase the final neutron flux to achieve BNCT applicable yield. This was taken into account before the moderation part for the selection of materials. 


\section{Discussion and results}

The enrichment of the thermal and epithermal components of the beam and the corresponding formation of the flow must be performed using a Beam Shaping Assembly (BSA). The main goal of this work is the design of the BSA using the software package GEANT4, which will be useful for medical purposes, particularly for BNCT.

First, the BSA should be a composition of carefully selected moderators to reduce the neutron energy to the thermal/epithermal range. Second, for an effective treatment, it is necessary to use reflectors, collimators and shielding materials to have a proper focusing of the quasi-monochromatic neutron flux, which will also satisfy the IAEA's criteria.

The therapeutic dose of ${ }^{10} \mathrm{~B}$ isotope is greater or equal than $20 \mu \mathrm{g}$ per gram of tumor tissue or $10^{9}$ of ${ }^{10} \mathrm{~B}$ per cell. At the same time, thermal/epithermal neutron flux must be high enough to be effectively captured by each ${ }^{10} \mathrm{~B}$ located inside of the cell, as the main goal of BNCT is to kill the tumor cell by cell.

Thermalization of neutrons could be done by using layers of different types of materials when each layer could be applied for neutrons in the exact energetic range. At the same time, the number of neutrons should not be low or non-applicable for BNCT.

Natural molybdenum with optimal thickness $20 \mathrm{~cm}$, installed as the first layer of BSA, works as a multiplier. With $(n, 2 n)$ nuclear reactions, it is possible to decrease the energy of neutrons and at the same time get a higher amount of thermal/epithermal flux. Due to earlier studies [15], it may be admitted that molybdenum is an optimal material for multiplication of neutron yield and at the same time for thermalization on the base $(n, \mathrm{x} n)$ nuclear reactions.

Right after the ${ }^{\text {nat }} \mathrm{Mo}$, there are installed $45-\mathrm{cm}$-thick iron and $45-\mathrm{cm}$-thick paraffin boric acid to decrease the energy of neutrons as much as possible. Iron is a suitable material to decrease the energy of neutrons down to $1 \mathrm{MeV}$, and the other important moderator is a mix of paraffin and boric acid to make the flux thermal/epithermal.

Moderators should be covered by the reflector to focus the beam. In this case, 20-cm-thick $\mathrm{Pb}$ is used as a reflector, which is known as one of the useful materials and widely used as an optimal one [16-19].

In this GEANT4 model, the whole BSA system was covered by thick concrete from sides. It was considered that the BSA should be installed in the wall, which will separate the room for patients from the rest of the experimental hall. The created BSA gives the possibility to achieve neutron flux, which is suitable with the International Atomic Energy Agency (IAEA) recommendations. Fig. 2 presents the energy distribution of neutrons when BSA consisted of 20-cm-thick Mo, 45-cm-thick Fe and 45-cm-thick paraffin boric acid. As a result of GEANT4 simulations, the registered neutron flux is around $0.968 \times 10^{9} \mathrm{n} / \mathrm{s} / \mathrm{cm}^{2}$ from $6.24 \times 10^{14}$ proton/s (when the current is $100 \mu \mathrm{A}$ ) and consisted of $69 \%$ epithermal neutrons, around $17 \%$ fast neutrons, and thermal neutrons amount is just $14 \%$. Experimentally the results should be proven at A. Alikhanian National Science Laboratory, where the Cyclone 18/18 is installed. Compared with previous result [14], where the BSA consisted from 5-cm-thick Bi, 55-cm-thick Fe, 10 -cm-thick Al, 5-cm-thick graphite and 10 -cm-thick ${ }^{7} \mathrm{LiF}$ as moderators and $10-\mathrm{cm}$-thick $\mathrm{Pb}$ as reflector surrounding the moderation system, the total flux was 0.96 $\times 10^{6} \mathrm{n} / \mathrm{s} / \mathrm{cm}^{2}$ from $6.24 \times 10^{14}$ proton/s and only $12 \%$ of neutrons were in the energetic range of up to $10 \mathrm{keV}$, while the rest were fast neutrons.

To apply neutron flux for medical purposes or for BNCT, there is a necessity to study epithermal neutrons with energies up to $10 \mathrm{keV}$, which loses energy because of tissue penetration and becomes thermalized [20]. Fig. 3a, b presents the aforementioned energetic range neutrons, which was possible to achieve by using BSA consisted from 20 -cm-thick Mo neu- 
Fig. 2 Energy distribution of neutrons after the BSA
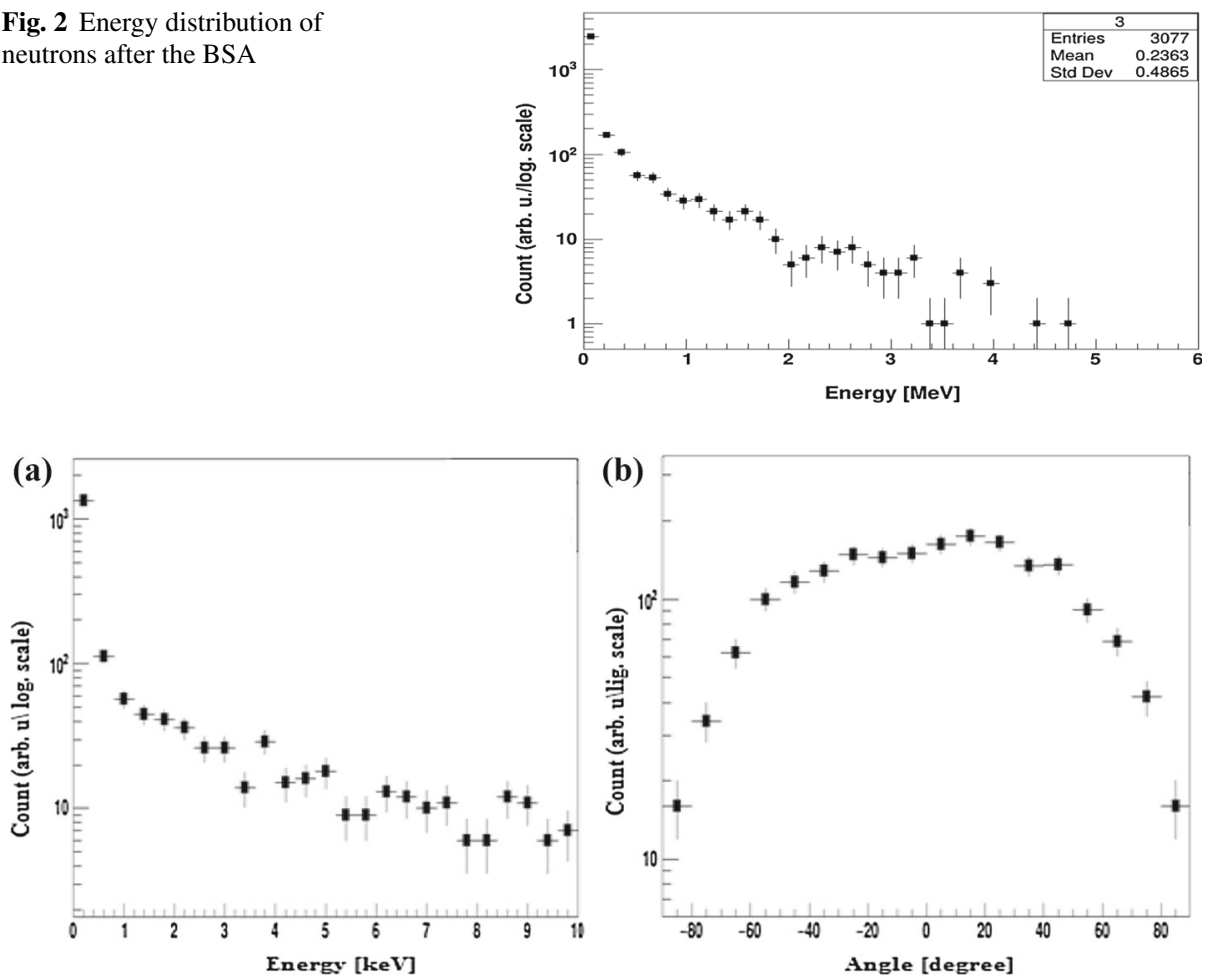

Fig. 3 Energy (a) and angular (b) distribution of up to $10 \mathrm{keV}$ neutrons

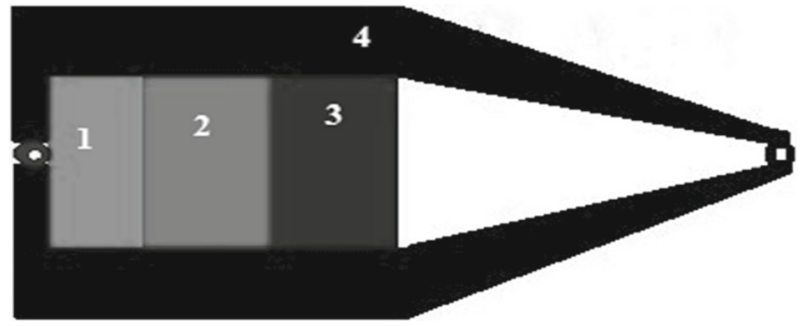

Fig. 4 BSA schema consisted from 20-cm-thick Mo (1), $45 \mathrm{~cm}$ Fe (2) and $45 \mathrm{~cm}$ Paraffin Boric Acid (3) as moderators, and 20-cm-thick $\mathrm{Pb}(4)$ as reflector

tron multiplier, $45 \mathrm{~cm} \mathrm{Fe}$ and $45 \mathrm{~cm}$ paraffin boric acid as moderators, and 20-cm-thick $\mathrm{Pb}$ as reflector/collimator (Fig. 4).

Because of the application for medical purposes, the total dose includes not only the influence of neutrons, but also gamma rays. Fig. 5a presents energy distribution of gamma rays, and almost $99.4 \%$ of gammas have energies up to $6 \mathrm{MeV}$. It is known that even $6 \mathrm{MeV}$ monochromatic gamma ray flux is useful for radiotherapy [21-23].

The statistical error for the registered gamma rays from 7 to $9 \mathrm{MeV}$ increased from 22 to $100 \%$. In this case, $0.6 \%$ of gamma rays, which are in the aforementioned energetic region, can be even less. Nevertheless, the estimation of gamma rays influence and its contribution on the total dose should be done in greater detail in the future, but as a result of simulations the 

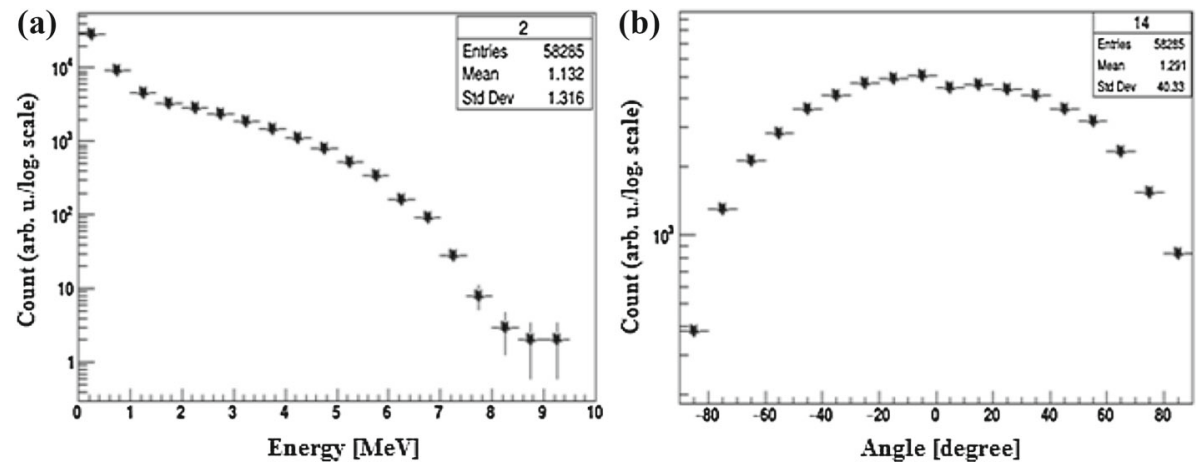

Fig. 5 Energy (a) and angular (b) distribution of gamma rays

registered flux is $1.083 \times 10^{10} \mathrm{gamma} / \mathrm{s} / \mathrm{cm}^{2}$ from the highest possible proton current, which is $100 \mu \mathrm{A}$ or around $6.24 \times 10^{14}$ proton/s. It is important to take into account the focusing of gamma rays as well. Figure $5 \mathrm{~b}$ is the angular distribution, which shows that gamma flux is mainly focused on the central part of the surface of the register/detector. The aforementioned fact and the appropriate figure also show that the reflector of a BSA focuses not only neutrons but gamma rays too. In the case of BNCT, the impact of gamma rays and fast neutrons should be suppressed. It is worth to be mentioned that the capturing process of neutrons by boron nucleus usually followed by $0.478 \mathrm{MeV}$ gamma rays, which should be detected, for example by SPECT. Due to the existence of gamma rays emitted from the target, as well as, from other components of the BSA, it will make technical difficulties and impede the detection of gamma rays from the capturing process. It is known that $\mathrm{Pb}$ is one of the widely used gamma ray filters [24-26], but its influence on epithermal neutrons and on the final quality of the neutron beam should be estimated only after deeply detailed investigations.

\section{Summary}

The subject of this work has been the simulation study by GEANT4 program to create a model of BSA, which will thermalize neutrons and make them applicable for BNCT. The comparison of neutron fluxes obtained previously by different types of BSA, with the latest one, consisted of 20-cm-thick molybdenum, 45-cm-thick iron and 45-cm-thick paraffin boric acid layers as moderators and 20 -cm-thick lead as reflector, makes possible to achieve $0.968 \times 10^{9} \mathrm{n} / \mathrm{s} / \mathrm{cm}^{2}$ flux of neutrons from $6.24 \times 10^{14}$ proton/s. The suitability with IAEA recommendations is quite high. It is worth to be mentioned that the necessary amount of neutrons was possible to obtain, besides that, around $70 \%$ of neutrons are epithermal, which is also an important achievement. The shielding problems due to the backscattered neutrons, as well as fast neutrons and gamma rays, influence together with total dose calculations, which is a matter of later research, and will be done in the nearest future after deeply detailed investigations.

Data availability statement This manuscript has associated data in a data repository. [Authors' comment: All data of the manuscript are available publicly upon request by contacting the corresponding author.]

Open Access This article is licensed under a Creative Commons Attribution 4.0 International License, which permits use, sharing, adaptation, distribution and reproduction in any medium or format, as long as you give appropriate credit to the original author(s) and the source, provide a link to the Creative Commons licence, 
and indicate if changes were made. The images or other third party material in this article are included in the article's Creative Commons licence, unless indicated otherwise in a credit line to the material. If material is not included in the article's Creative Commons licence and your intended use is not permitted by statutory regulation or exceeds the permitted use, you will need to obtain permission directly from the copyright holder. To view a copy of this licence, visit http://creativecommons.org/licenses/by/4.0/.

\section{References}

1. R.F. Barth, J.A. Coderre, M.G. Vicente, T.E. Blue, Boron neutron capture therapy of cancer: current status and future prospects. Clin. Cancer Res. 11, 3987-4002 (2005)

2. D.N. Slatkin, A history of boron neutron capture therapy of brain tumors. Postulation of a brain radiation dose tolerance limit. Brain 114, 1609-1629 (1991)

3. R.F. Barth, Boron neutron capture therapy: a critical assessment. J. Neurooncol. 62, 1-210 (2003)

4. T. Aihara, J. Hiratsuka, N. Morita, M. Uno, Y. Sakurai, A. Maruhashi, K. Ono, Harada T first clinical case of boron neutron capture therapy for head and neck malignancies using 18 F-BPA PET. Head Neck 28, 850-855 (2006)

5. I. Kato, K. Ono, Y. Sakurai, M. Ohmae, A. Maruhashi, Y. Imahori, M. Kirihata, M. Nakazawa, Y. Yura, Effectiveness of BNCT for recurrent head and neck malignancies. Appl. Radiat. Isot. 61, 1069-1073 (2004)

6. I. Kato, Y. Fujita, A. Maruhashi, H. Kumada, M. Ohmae, M. Kirihata, Y. Imahori, M. Suzuki, Y. Sakrai, T. Sumi et al., Effectiveness of boron neutron capture therapy for recurrent head and neck malignancies. Appl. Radiat. Isot. 67, S37-S42 (2009)

7. L. Kankaanranta, T. Seppälä, H. Koivunoro, K. Saarilahti, T. Atula, J. Collan, E. Salli, M. Kortesniemi, J. Uusi-Simola, P. Valimaki et al., Boron neutron capture therapy in the treatment of locally recurred head-and-neck cancer: final analysis of a phase I/II trial. Int. J. Radiat. Oncol. Biol. Phys. 82, e67-e75 (2012)

8. T.E. Blue, J.C. Yanch, Accelerator-based epithermal neutron sources for boron neutron capture therapy of brain tumors. J. Neurooncol. 62, 19-31 (2003)

9. Cyclone 18/9 Standard and High Current model, Product descrip, IBA Molecular, C189HC-TPD-R09, July (2007). https://www.iba-radiopharmasolutions.com

10. IBA Cyclone 18 Moving ahead delivering more. https://www.researchgate.net/publication/338531235_ CYCLONE_R_18_MOVING_AHEAD_DELIVERING_MORE

11. Current status of neutron capture therapy. IAEA-TECDOC-1223, International Atomic Energy Agency (2001). https://pub.iaea.org/MTCD/Publications/PDF/te_1223_prn.pdf

12. J.G. Fantidis, E. Saitioti, D.V. Bandekas, N. Vordos, Optimised BNCT facilitybased on a compact D-D neutron generator. Int. J. Radiat. Res. 11, 4 (2013)

13. Geant4 Collaboration, Book For Application Developers (Release 10.4), Rev1.0: 8 Dec 2017. https:// GEANT4.web.cern.ch/support

14. R. Avagyan, R. Avetisyan, V. Ivanyan, I. Kerobyan, GEANT4 simulations of a beam shaping assembly design and optimization for thermal/epithermal neutrons. Acta Phys. Pol. B 48, 10 (2017)

15. K. Shibata, A. Ichihara, S. Kunieda, Calculation of neutron nuclear data on molybdenum isotopes for JENDL-4. J. Nucl. Sci. Technol. 46(3), 278-288 (2009)

16. A.A. Burlon, A.J. Kreiner, S.M. White, B. Blackburn, J.C. Yanch, Med. Phys. 28, 796 (2001)

17. F.S. Rasouli, S.F. Masoudi, Y. Kasezas, Ann. Nucl. Energy 39, 18 (2012)

18. H. Koivunoro, I. Auterinen, A. Kosunen, P. Kotiluoto, T. Seppälä, S. Savolainen, Phys. Med. Biol. 48, 291 (2004)

19. R. Avagyan, R. Avetisyan, V. Ivanyan, I. Kerobyan, Nucl. Instrum. Methods Phys. Res. B 402, 247 (2017)

20. R.F. Barth, Z. Zhang, T. Liu, A realistic appraisal of boron neutron capture therapy as a cancer treatment modality. Cancer Commun. (Lond) 38, 36 (2018)

21. E.B. Podgorsak, Radiation Oncology Physics: A Handbook for Teachers and Students (IAEA, Vienna, 2005)

22. M.J. Gazda, L.R. Coia, Principles of radiation therapy, in Cancer Management: A Multidisciplinary Approach: Medical, Surgical and Radiation Oncology, ed. by R. Pazdur, L.R. Coia, W.J. Hosians, L.D. Wagman (PRR Inc, Melville, 2001), pp. 9-19

23. G.D. Grant, Radiation therapy physics. Johns Hopkins APL Tech. Dig. 4, 1 (1983)

24. M. Asnal, T. Liamsuwan, T. Onjun, An evaluation on the design of beam shaping assembly based on the D-T reaction for BNCT. J. Phys. Conf. Ser. 611, 012031 (2015) 
25. H. Miyamaru, I. Murata, Neutron and gamma-ray dose evaluation on accelerator neutron source using p-Li reaction for BNCT. Prog. Nucl. Sci. Technol. 1, 533-536 (2011)

26. R. Avagyan, R. Avetisyan, V. Ivanyan, I. Kerobyan, Calculations of neutron yield and gamma rays intensity by GEANT4. Armen. J. Phys. 9(4), 315-323 (2016) 